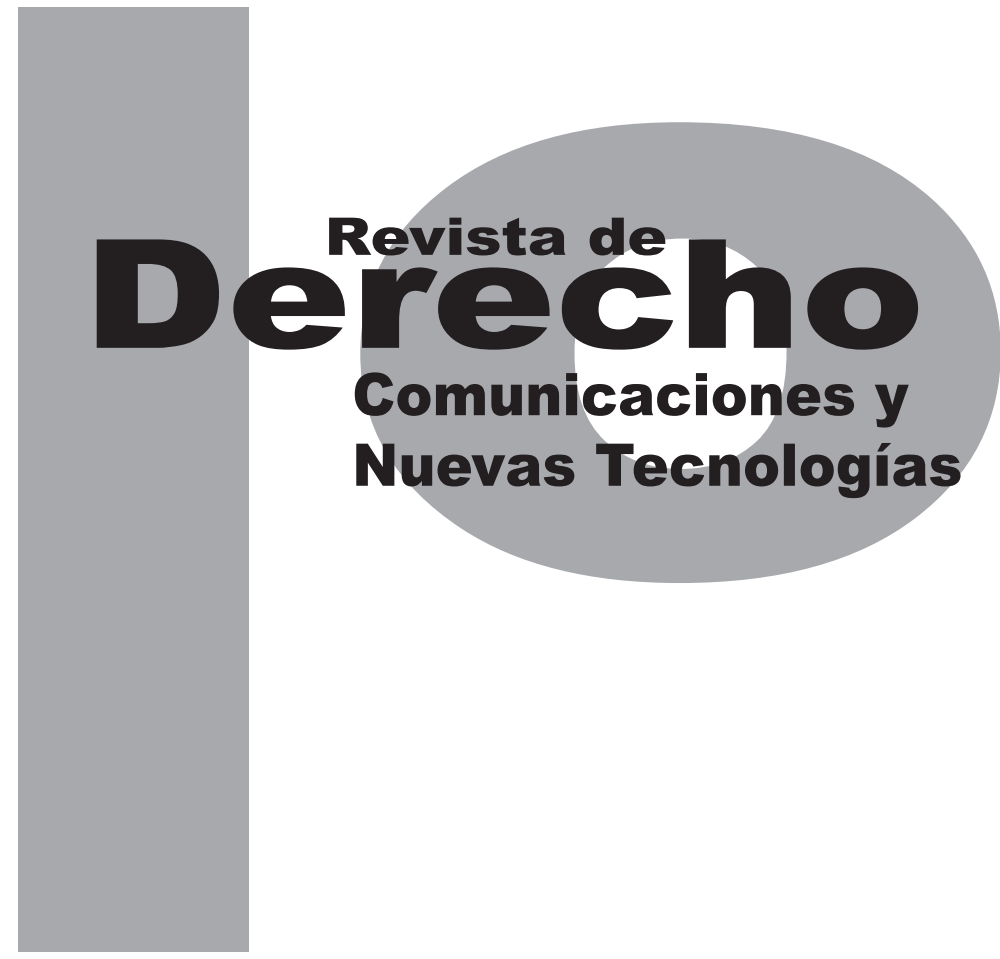

\title{
LA EXPLORACIÓN EN EL ESPACIO: PRINCIPIO DE COOPERACIÓN
}

\author{
LAURA OSORIO \\ ANDRÉS UMAÑA \\ Artículo de reflexión \\ DOI: http://dx.doi.org/10.15425/redecom.12.2014.07
}

Universidad de los Andes
Facultad de Derecho

Revista de Derecho, Comunicaciones y Nuevas Tecnologías

No.12, Julio - Diciembre de 2014. ISSN 1909-7786 


\section{La exploración en el espacio: principio de cooperación}

\section{Resumen}

El presente ensayo analiza el régimen jurídico que envuelve a la cooperación internacional en el ámbito del espacio ultraterrestre, así como su exploración. Pretende mostrar los diferentes tipos de cooperación, y cómo han sido desarrollados históricamente por las necesidades de los países involucrados en la creación del derecho del espacio ultraterrestre y sus efectos posteriores. Para esto se revisan la doctrina y los diferentes tratados y resoluciones señalados por las Naciones Unidas como de vital importancia para el presente tema. Adicionalmente, se busca recalcar los problemas que este tipo de evolución jurídica ha conllevado y unas posibles soluciones a estos, que permitan la subsistencia de la exploración del espacio a través del principio de la cooperación internacional.

Palabras clave: Espacio ultraterrestre, cooperación internacional, exploración, consenso, confianza, equidad, estación espacial, NASA, JAXA, ASI, ESA.

\section{The exploration of outer space: Principle of cooperation}

\section{Abstract}

This paper analyzes the legal regime that involves international cooperation in the field of outer space, regarding its exploration. It aims to show the different types of cooperation, as they have been historically developed for the needs of the countries involved in the creation of the Law of Outer Space and its aftermath. To do this, this paper bases its content in doctrine and the various treaties and resolutions identified by the United Nations as of vital importance for this issue. Additionally, it draws attention to the problems that this type of legal developments has led and some possible solutions to these, which assures the space exploration through the principle of international cooperation.

Keywords: Outer space, international cooperation, exploration, consensus, trust, equity, space station, NASA, JAXA, CSA, ESA.

\section{A exploração no espaço: princípio de cooperação}

\section{Resumo}

O presente ensaio analisa o regime jurídico que envolve a cooperação internacional no âmbito do espaço ultraterrestre, assim como sua exploração. Pretende mostrar os diferentes tipos de cooperação, e como têm sido desenvolvidos historicamente pelas necessidades dos países envolvidos na criação do direito ao espaço ultraterrestre e seus efeitos posteriores. Para isto são revisados a doutrina e os diferentes tratados e resoluções assinalados pelas Nações Unidas como de vital importância para o presente tema. Adicionalmente, busca-se recalcar os problemas que este tipo de evolução jurídica implicou e umas possíveis soluções a estes, que permitam a subsistência da exploração do espaço através do princípio da cooperação internacional.

Palavras-chave: espaço ultraterrestre, cooperação internacional, exploração, consenso, confiança, equidade, estação espacial, NASA, JAXA, ASI, ESA. 


\title{
La exploración en el espacio: principio de cooperación*
}

\author{
Laura Osorio ** \\ Andrés Umaña***
}

\section{SUMARIO}

I. CAPITULO I - A. Introducción - B. ¿Cuáles son los principios del derecho del espacio? - 1. ¿Qué es el principio de cooperación? - C. Tratados y resoluciones internacionales que reafirman el principio de cooperación - D. La exploración en el espacio ultraterrestre bajo el principio de cooperación - E. El ejemplo de la cooperación internacional en la Estación Espacial Internacional MIR - II. CAPITULO II - A. Exploración en el espacio - 1. Régimen Jurídico - 2. Análisis Económico - 3. Problemas del derecho del espacio en el siglo XXI - B. Propuesta. Soluciones para la crisis del derecho del espacio - 1. Reforma al consenso - 2. Fondo del espacio - 3. Subcomisión verificadora - III. CONCLUSIONES - Referencias

* $\quad$ Cómo citar este artículo: Osorio, L. y Umaña, A. (Diciembre, 2014). La exploración en el espacio: principio de cooperación. Revista de Derecho, Comunicaciones y Nuevas Tecnologías, 12. Universidad de los Andes (Colombia).

** Laura Osorio es estudiante de séptimo semestre de Derecho de la Universidad de los Andes. Correo electrónico: I.osorio151@uniandes.edu.co

*** Andrés Umaña es estudiante de octavo semestre de Derecho de la Universidad de los Andes y graduado de Advanced Social, Economic and Political Studies del programa del Phoenix Institute en la Universidad de Notre Dame. Correo electrónico: a.umana41@uniandes.edu.co 


\section{CAPÍTULO I}

\section{A. Introducción}

El inicio de la Guerra Fría vino acompañado de una serie de progresos tecnológicos y descubrimientos científicos motivados, precisamente, por la competencia entre los Estados Unidos de América y la Unión Soviética, que intentaban demostrar el poderío y potencial armamentístico de cada nación. Esto, a su vez, hizo posible la exploración del espacio ultraterrestre, que empezó en 1957 con el lanzamiento del primer satélite hecho por el hombre, el Sputnik I. Este avance marcó un cambio en la manera en que se percibía el universo. Debido a esto, y para evitar una inminente confrontación en el espacio ultraterrestre, se empezó a hablar de la necesidad de crear un conjunto de normas, que fueran en sí mismas una rama del derecho internacional, ya que

la utilización del espacio exterior estaba situado fuera de la jurisdicción nacional de los Estados. Su desarrollo se liga fuertemente al avance tecnológico y su misión sería regular las actividades de los Estados, las organizaciones internacionales intergubernamentales y empresas privadas [...]. El objeto principal de esta nueva jurisdicción era el beneficio de todos los pueblos y el desarrollo de un carácter exclusivamente pacífico que debe revestir dicha actividad. (Williams, 1990, p. 9, cursivas fuera del texto)

A partir de esto fue posible la creación del Copuos (siglas en inglés para el Comité para Usos Pacíficos del Espacio Extraatmosférico de las Naciones Unidas) en 1958.
En 1963, esta comisión permitió la firma de la primera resolución sobre el uso adecuado del espacio exterior. En este documento se establecieron una serie de principios rectores que dictan cómo debe ser el uso y la exploración adecuada del espacio ultraterrestre. Entre los principios que podemos encontrar está el de la cooperación internacional, que tiene como fin equilibrar las diferencias que existen entre los diferentes países mediante una serie de imposiciones que se establecen en dichas naciones. "La cooperación internacional siempre ha hecho referencia al trabajo en comunidad y a la unión de esfuerzos y recursos entre los diferentes países del mundo que deciden colaborar en la ejecución de proyectos y nuevas estrategias que, después de un tiempo de dedicación, les representan beneficios, sobre todo a nivel de desarrollo". No obstante, al tratarse de una cooperación en beneficio de toda la humanidad, se le debe dar especial atención a las necesidades de los países en vía de desarrollo. En materia de cooperación y asistencia mutua, son los mismos Estados los que determinan su grado de participación y las modalidades a través de las cuales quieren llevar a cabo dicha cooperación. Los países escogerán aquellas que "consideren más eficaces y adecuadas, incluidas, entre otras, la cooperación gubernamental y no gubernamental; comercial y no comercial; mundial, multilateral, regional o bilateral y la cooperación internacional entre países de distintos niveles de desarrollo -Asamblea General de las Naciones Unidas, 1997, art. 4-" (Valera, 2013, p. 9). Esto es posible gracias a tres factores, que permiten que los países se ayuden mutuamente: 
1. Consenso: Esto es, un acuerdo, en donde se aprueba una decisión siempre y cuando no haya ningún tipo de oposición. Es importante recordar que el consenso no implica una posición activa frente a la posición que los Estados toman, basta con que el país que no comparte la posición de los otros se adhiera al consenso sin oponerse. Este tipo de negociación, que lleva a un acuerdo, genera confianza frente al cumplimiento de la norma.

2. Confianza: Se refiere a la confianza en el cumplimiento de la norma por parte de los demás Estados. Siendo así, como lo indica Valera:

Una herramienta para reducir el temor $y$ además de esto, hay que considerar que hay ciertas características propias del espacio ultraterrestre que hacen que la confianza pase a ocupar un lugar protagónico. La alta capacidad de intrusión (sobre todo de las grandes potencias) y el hecho de que las naciones y la comunidad internacional, en general, dependan cada vez más de la tecnología espacial para lograr objetivos económicos y sociales en la Tierra, son circunstancias que reflejan la necesidad de evitar riesgos. (2003, p.9; cursivas fuera del texto)

3. Equidad: El concepto de equidad representa al trato de iguales entre iguales y de desiguales como desiguales. En otras palabras, es el reconocimiento de las diferencias que tienen los países. Por esto la norma del espacio ultraterrestre no puede ser igual para todos, dado que no siempre se está en las mismas condiciones, ya bien sea económicas o tecnológicas, que permitan el cumpli- miento de las obligaciones y requerimientos que se les suele imponer a los países más desarrollados.

Como podemos ver, estos elementos permiten la existencia de la cooperación porque motiva a los Estados a ayudarse entre sí, teniendo en cuenta que no todos están en la misma posición y que por lo tanto el tipo de cooperación debe variar dependiendo de cada situación.

Es a partir de esto que nos hemos propuesto, a través de este trabajo, investigar cuáles han sido algunos descubrimientos científicos hechos en torno al espacio ultraterrestre, y cómo estos han sido posibles gracias a la cooperación internacional. También estudiaremos los tratados y resoluciones claves para el desarrollo de estos conceptos. Sumado a esto, resaltaremos los problemas del derecho del espacio ultraterrestre internacional y cuáles son las posibles soluciones. Para poder hacer esto, empezaremos por señalar cuál es la normatividad legal aplicable, y explicar más detalladamente en qué consiste y cómo afecta el principio de cooperación a los diferentes Estados, siendo de esta manera un artículo de revisión del tema.

\section{B. ¿Cuáles son los principios del derecho del espacio?}

Con los avances tecnológicos y la materialización del sueño de poder, efectivamente, explorar el espacio ultraterrestre, gracias al lanzamiento del Sputnik I en 1957 se planteó la problemática jurídica del espacio. Esto ayudó a que al siguiente año, en 1958, la Unión Soviética y 
Ios Estados Unidos de América presionaran a la Asamblea General de las Naciones Unidas, con el fin de incluir el tema de la legislación espacial entre los temas que debían ser tratados (Seara, 1981, pp. 27 y 28). Esto llevó a que el 13 de diciembre de 1958 se creara la Comisión Especial sobre Utilizaciones Pacíficas del Espacio Ultraterrestre, que posteriormente sería reemplazada por la Comisión sobre la utilización del espacio ultraterrestre con fines pacíficos, la cual está constituida por dos subcomisiones, la Subcomisión de Asuntos Científicos y Técnicos y la Subcomisión de Asuntos Jurídicos.

El siguiente hito del derecho del espacio ultraterrestre lo constituyó en 1961 la afirmación de la Asamblea General de las Naciones Unidas respecto a que las normas del derecho internacional y la carta de las Naciones Unidas eran aplicables en el espacio exterior. A pesar de esto, no fue sino hasta 1963 cuando se expidió el primer documento relativo al uso del espacio, la Declaración de los principios jurídicos que deben regir las actividades de los Estados en la exploración y utilización del espacio ultraterrestre. En esta resolución se plantean nueve principios que deberían ser seguidos a la hora de explorar o utilizar el espacio exterior, y que a continuación se citan textualmente:

1. La exploración y la utilización del espacio ultraterrestre deberá hacerse en provecho y en interés de toda la humanidad.

2. El espacio ultraterrestre y los cuerpos celestes podrán ser libremente explorados y utilizados por todos los Estados en condiciones de igualdad y en conformidad con el derecho internacional.

3. El espacio ultraterrestre y los cuerpos celestes no podrán ser objeto de apropiación nacional mediante reivindicación de soberanía, mediante el uso y la ocupación, ni de ninguna otra manera.

4. Las actividades de los Estados en materia de exploración y utilización del espacio ultraterrestre deberán realizarse de conformidad con el derecho internacional, incluido la Carta de las Naciones Unidas, en interés del mantenimiento de la paz y la seguridad internacionales y del fomento de la cooperación y la comprensión internacionales.

5. Los Estados serán responsables internacionalmente de las actividades nacionales que realicen en el espacio ultraterrestre los organismos gubernamentales o las entidades no gubernamentales, así como de asegurar la observancia en la ejecución de esas actividades nacionales de los principios enunciados en la presente Declaración. Las actividades de entidades no gubernamentales en el espacio ultraterrestre deberán ser autorizadas y vigiladas constantemente por el Estado interesado. Cuando se trate de actividades que realicen en el espacio ultraterrestre una organización internacional, la responsabilidad en cuanto a la aplicación de los principios proclamados en la presente Declaración corresponderá a esa organización internacional y a los Estados que forman parte de ella. 
6. En la exploración y la utilización del espacio ultraterrestre, los Estados se guiarán por el principio de la cooperación y la asistencia mutua, y en todas sus actividades en el espacio ultraterrestre deberán tener debidamente en cuenta los intereses correspondientes de los demás Estados. Si un Estado tiene motivos para creer que una actividad o un experimento en el espacio ultraterrestre, proyectado por él o por sus nacionales, crearía un obstáculo capaz de perjudicar las actividades de otros Estados en materia de exploración y utilización del espacio ultraterrestre con fines pacíficos, celebrará las consultas internacionales oportunas antes de emprender esa actividad o ese experimento. Si un Estado tiene motivos para creer que una actividad o un experimento en el espacio ultraterrestre proyectado por otro Estado, crearían un obstáculo capaz de perjudicar las actividades en materia de exploración y utilización del espacio ultraterrestre con fines pacíficos, podrá pedir que se celebren consultas sobre esa actividad o ese experimento.

7. El Estado en cuyo registro figure el objeto lanzado al espacio ultraterrestre retendrá su jurisdicción y control sobre tal objeto, así como sobre todo el personal que vaya en él, mientras se encuentre en el espacio ultraterrestre. La propiedad de los objetos lanzados al espacio ultraterrestre y de sus partes componentes no se modificara con motivo de su paso por el espacio ultraterrestre ni de su regreso a la Tierra. Cuando esos objetos o esas partes componentes sean hallados fuera de los límites del Estado en cuyo registro figure, se devolverá a ese Estado, que deberá proporcionar, antes de que se efectúe la devolución, los datos de identificación que en su caso se soliciten.

8. Todo Estado que lance u ocasione el lanzamiento de un objeto al espacio ultraterrestre, y todo Estado desde cuyo territorio o cuyas instalaciones se lance un objeto, será responsable internacionalmente de los daños causados a otro Estado extranjero o a sus partes componentes en la Tierra, en el espacio aéreo o en el espacio ultraterrestre.

9. Los Estados considerarán a todos los astronautas como enviados de la humanidad en el espacio ultraterrestre, y les prestarán toda ayuda posible en caso de accidente, peligro o aterrizaje forzoso en el territorio de un Estado extranjero o en alta mar. Los astronautas que hagan dicho aterrizaje serán devueltos por medio seguro y sin tardanza al Estado de registro de su vehículo espacial.

Dado que este documento no tenía un valor de hard law, por tratarse de una resolución, en el año 1967 se consolidó lo expuesto por la Declaración de 1963 por medio de un tratado (Tratado sobre los principios que deben regir las actividades de los Estados en la exploración y utilización del espacio ultraterrestre, incluso la Luna y otros cuerpos celestes). El Tratado de 1967, como lo afirma Nicolás Sussman en su trabajo de tesis, El Tratado de 1967: la extensión y garantía del mantenimiento de la paz y la seguridad internacional en el espacio, tiene 
una serie de artículos dedicados a la prevención de riesgos que cobran fuerza gracias a los principios de la no apropiación, el uso pacífico del espacio ultraterrestre y de la cooperación. Principios que ya habían sido tratados en la Declaración de 1963, como lo podemos ver en los puntos III, IV y VI. Es precisamente por la fuerza vinculante emanada del tratado de 1967 y de su establecimiento como base para el desarrollo del espacio en la declaración de 1963 que estos tres principios se vuelven supremamente importantes tanto para la legislación del espacio ultraterrestre, como para su uso y exploración.

Debido a lo anterior y para un mayor entendimiento del presente trabajo proseguiremos por explicar qué es el principio de cooperación.

\section{1. ¿Qué es el principio de cooperación?}

En el derecho internacional, el principio de cooperación ha sido ampliamente utilizado y desarrollado, por lo que no basta con simplemente definirlo como el intento de equilibrar las diferencias existentes entre países mediante una serie de ayudas o imposiciones hechas a diferentes naciones.

Una definición más exacta que podemos usar, es la dada por la Universidad del Rosario, la cual afirma que

La cooperación internacional es una herramienta de colaboración que apoya procesos de desarrollo mediante la transferencia de recursos técnicos y financieros entre diversos actores del sistema internacional (gobiernos, entes territoriales, organizaciones de la sociedad ci- vil, ONG) [...]. Un segundo tipo de cooperación sería la cooperación descentralizada, cuyo enfoque se caracteriza por impulsar el desarrollo desde las entidades territoriales y la sociedad civil, como nuevos actores que realizan propuestas para la cooperación y que tienen la capacidad para llevarlas a cabo. El concepto de cooperación descentralizada fue planteado por la Unión Europea como una iniciativa para fomentar un enfoque participativo en la cooperación y un papel activo de los agentes locales de los países del sur en sus procesos de desarrollo. (Universidad del Rosario).

Estos conceptos han sido ampliados, como lo podemos ver en situaciones como la del fallo de la Corte Internacional de Justicia en el caso del Estrecho de Corfú (CIJ, 1949). Por este motivo, se ha llegado a diferenciar qué tipos de cooperación existen a nivel internacional:

1. Cooperación financiera: Puede ser reembolsable o no reembolsable. En los dos casos se asigna un recurso a un país, la diferencia recae en si este es un préstamo con una "facilidad", como amplios plazos de pago, o intereses bajos (reembolsables), o por el contrario, que estos nunca tengan que ser devueltos (no reembolsables).

2. Cooperación técnica: "Se lleva a cabo mediante la transferencia de conocimientos, habilidades y experiencias por parte de países u organizaciones con un nivel de desarrollo más avanzado con el fin de contribuir con el fortalecimiento de las capacidades, el avance tecnológico y la formación de los recursos humanos del beneficiario, entre otros" (Universidad del Rosario). 
3. Cooperación Técnica entre Países en Desarrollo (CTPD) o cooperación sur-sur: Esta consiste en el intercambio de experiencias y conocimientos técnicos entre naciones de igual nivel de desarrollo, con el fin de adaptarlo a su respectiva situación.

4. Cooperación triangular: Este tipo de cooperación es muy similar al de la CTPD, con la diferencia de que se trata de países en vía de desarrollo, y que este intercambio, como tal, es financiado por un tercer país (desarrollado).

5. Cooperación descentralizada: Esta "promueve el desarrollo desde el ámbito territorial. Esto quiere decir que las actividades de colaboración no se deben realizar necesariamente entre gobiernos nacionales, sino también entre administraciones subestatales y organizaciones de la sociedad civil".

6. Ayuda humanitaria y de emergencia: Cuando hay una situación de emergencia, ya bien sea por un conflicto armado o una catástrofe natural, se dan diferentes tipos de ayudas por parte de países u organizaciones, con el objetivo de salvaguardar a la población afectada. Este tipo de colaboración solo es posible si el país afectado no puede lidiar con el desastre por su propia cuenta o si se trata de un evento considerado como inusual. Es importante recalcar que este tipo de cooperación también puede ser dada con el fin de prevenir la emergencia.

7. Ayuda alimentaria: Este tipo de colaboración suele estar ligado a la ayuda humanitaria y de emergencia, aunque esto no siempre es el caso. Básicamente consiste en la donación o venta con "porcentajes condonables o créditos blandos", de productos alimenticios a poblaciones que por diferentes motivos no pueden obtener los alimentos necesarios para su supervivencia.

8. Cooperación cultural: Consiste en la asignación de recursos, con el fin de apoyar el desarrollo cultural, su fortalecimiento y su protección. Suele ser llevado a cabo por museos, grupos culturales y los gobiernos interesados en la preservación de la diversidad cultural.

9. Cooperación por becas: "Las becas son utilizadas para capacitar funcionarios, técnicos e investigadores en países más desarrollados, para que puedan desempeñar una función importante en sus países de origen gracias a los conocimientos y capacidades adquiridos". Estas se suelen dar por parte de entidades gubernamentales, por ejemplo, en algunos cursos de entrenamiento militar en Estados Unidos se suele capacitar a algunos militares colombianos.

Teniendo esto claro, podemos afirmar que se buscan varios tipos de cooperación en lo referente al espacio ultraterrestre, como se puede ver a través de múltiples tratados. Es evidente que se intenta que haya cooperación financiera, técnica, CTPD y descentralizada para el desarrollo de los programas espaciales. A su vez, se intenta que haya cooperación de ayuda humanitaria y de emergencia, en lo que respecta al rescate de objetos espaciales, de astronautas y 
en cuanto a compartir información relevante obtenida de los satélites de teleobservación (este tipo de colaboración, frente a la información que se puede obtener de la exploración del espacio, no solo se da por razones relevantes para prevenir situaciones de emergencia, sino también para el desarrollo de la humanidad, por esto, también se puede hablar de cooperación técnica, cuando se trate de compartir datos obtenidos del estudio y exploración del espacio ultraterrestre). Esto siempre se hace bajo las bases de la equidad, confianza y consenso, como ya se explicó anteriormente.

\section{Tratados y resoluciones internacionales que reafirman el principio de cooperación}

Como ya explicamos, el principio de cooperación ha sido ampliamente utilizado no sólo a través del derecho internacional, sino también del derecho del espacio ultraterrestre. Por este motivo, nombraremos los tratados y resoluciones que hacen provecho de este principio para el desarrollo de sus artículos. Para hacer esto, nos centraremos en los tratados y resoluciones que las mismas Naciones Unidas han calificado como poseedoras de un contenido importante sobre cooperación internacional. Esto lo hacemos con el fin de limitar el contenido, puesto que de otra forma tomaría demasiado tiempo señalar cuáles son los elementos de cooperación en todos los tratados y resoluciones, ya que aunque todos los tratados de derecho del espacio ultraterrestre tienen como base el principio de cooperación internacional, no todos lo desarrollan a fondo a través de sus artículos.
De acuerdo con la página oficial de las Naciones Unidas, ${ }^{1}$ los tratados de cooperación internacional relevantes para el derecho del espacio ultraterrestre son:

1. Tratado sobre los principios que deben regir las actividades de los Estados en la exploración y utilización del espacio ultraterrestre, incluso la Luna y otros cuerpos celestes (19 de diciembre de 1966).

2. Acuerdo sobre el salvamento y la devolución de astronautas y la restitución de objetos lanzados al espacio ultraterrestre (19 de diciembre de 1967).

3. Resoluciones 1721 A y B (XVI) de la Asamblea General, del 20 de diciembre de 1961. Cooperación internacional para la utilización del espacio ultraterrestre con fines pacíficos.

4. Acuerdo que debe regir las actividades de los Estados en la Luna y otros cuerpos celestes (5 de diciembre de 1979).

5. Principios que han de regir la utilización por los Estados de satélites artificiales de la Tierra para las transmisiones internacionales directas por televisión (10 de diciembre de 1982).

6. Principios relativos a la teleobservación de la Tierra desde el espacio (3 de diciembre de 1986).

Recuperado de http://www.un.org/spanish/documents/instruments/ docs_subj_sp.asp?subj=26 
7. Declaración sobre la cooperación internacional en la exploración y utilización del espacio ultraterrestre en beneficio e interés de todos los Estados, teniendo especialmente en cuenta las necesidades de los países en desarrollo (13 de diciembre de 1996).

Dado que ya hemos explicado la importancia para el principio de la colaboración del Tratado sobre los principios que deben regir las actividades de los Estados en la exploración y utilización del espacio ultraterrestre, incluso la Luna y otros cuerpos celestes, continuaremos explicando cuál ha sido el particular valor de cada uno de los tratados y declaraciones aquí presentes.

Respecto al Acuerdo sobre el salvamento y la devolución de astronautas y la restitución de objetos lanzados al espacio ultraterrestre, podemos decir que está fundado en el mismo principio de cooperación, ya que al consagrar la obligación de los Estados de prestar ayuda en situación de peligro, lo que se está esperando es precisamente un tipo de cooperación directa, con el fin de rescatar el objeto espacial o al astronauta y de devolverlos a sus respectivos países, aunque estos sean enemigos. Esto es claro, dado que el mismo tratado fue creado en plena Guerra Fría, obligando así a países encontrados, como lo eran los Estados Unidos de América y la Unión Soviética a cooperar entre ellos en este tipo de situaciones riesgosas (en una gran medida por la necesidad de proteger la información obtenida por los objetos espaciales o por los mismos astronautas).

Frente a la Resoluciones 1721 A y B (XVI) de la Asamblea General, del 20 de diciembre de
1961, sobre la Cooperación internacional para la utilización del espacio ultraterrestre con fines pacíficos, cabe resaltar que en esta se establece la necesidad de hacer que las Naciones Unidas fueran el foco central de la cooperación internacional "en materia de exploración y utilización del espacio ultraterrestre con fines pacíficos". También establece que todo beneficio obtenido de la exploración espacial debe hacerse extensivo a todos los Estados, y pide "el intercambio más amplio posible de información en esta esfera, y la ampliación de los programas internacionales relacionados con las aplicaciones prácticas de la tecnología y el desarrollo", promoviendo así la máxima cooperación en materia científica del espacio ultraterrestre.

El Acuerdo que debe regir las actividades de los Estados en la Luna y otros cuerpos celestes establece que cualquier actividad que sea conducida en la Luna u otros cuerpos celestes deberá ser hecha bajo el principio de cooperación. Esto se hace con el fin de garantizar la confianza entre los países, perpetuando la paz en el espacio y así cumpliendo con otro objetivo del derecho del espacio ultraterrestre (Naciones Unidas, 1966). El elemento de la cooperación es de vital importancia en este tratado, ya que promueve no solo la paz, sino también el desarrollo de los conocimientos científicos sobre el universo, y la adecuada exploración del espacio, por medio de la asistencia mutua, persiguiendo así el conocimiento universal sobre temas que de otra forma serían información privada de cada Estado.

Cuando se estudia la resolución de los Principios que han de regir la utilización por los Es- 
tados de satélites artificiales de la Tierra para las transmisiones internacionales directas por televisión, se puede ver que entre los objetivos se encuentra el del "intercambio mutuo de información y conocimientos en las esferas de la cultura y de la ciencia, contribuir al desarrollo educativo, social y económico, especialmente de los países en desarrollo, elevar la calidad de vida de todos los pueblos y proporcionar esparcimiento con el debido respeto a la integridad política y cultural de los Estados" (Naciones Unidas, 1982). Este objetivo solamente puede llegar a ser alcanzado a través de la cooperación, haciendo que este principio sea esencial para la misma existencia de la resolución. Adicionalmente, cabe mencionar que en esta se explica la necesidad de tener consultas entre los Estados para garantizar el préstamo y uso adecuado de diferentes señales, siendo esto otra forma de reforzar el principio de cooperación en el derecho ultraterrestre.

La resolución de los Principios relativos a la teleobservación de la Tierra desde el espacio usa y refuerza el principio de cooperación. Esto sucede ya que obliga a los Estados a compartir la información obtenida por sus satélites de teleobservación. A su vez, determina una serie de condiciones para este tipo de colaboración, reglamentándola de tal manera que la información dada sea pagada por un precio razonable. Del mismo modo, instaura la obligación de cooperar más allá de cualquier tipo de negociación frente a la necesidad de proteger a la humanidad de daños que se puedan presentar y prever gracias a este tipo de tecnología.
La Declaración sobre la cooperación internacional en la exploración y utilización del espacio ultraterrestre en beneficio e interés de todos los Estados, teniendo especialmente en cuenta las necesidades de los países en desarrollo, empieza por afirmar que la cooperación se debe llevar a cabo de acuerdo con lo impuesto por el derecho internacional, recalcando que "la cooperación [...] se realizará en beneficio e interés de todos los Estados, sea cual fuere su grado de desarrollo económico, social, científico o técnico, e incumbirá a toda la humanidad. Deberán tenerse en cuenta especialmente las necesidades de los países en desarrollo" (Naciones Unidas, 1996). Adicionalmente, la declaración indica que los Estados podrán desarrollar como deseen el principio de cooperación y los contratos o negociaciones que sean conjuntas a esto. A pesar de lo anterior, en este documento se especifica que esta libertad tiene como límites la equidad, la razonabilidad y la necesidad de proteger a la humanidad. ${ }^{2}$

También menciona la obligación de los países de ayudar en el desarrollo de la capacidad técnica y de los programas del espacio ultraterrestre, adecuados para cada nación, bien sea cooperación "comercial y no comercial; mundial, multilateral, regional o bilateral", teniendo en cuenta sus necesidades, reforzando de esta manera el mismo concepto de equidad. Teniendo claro esto, se prosigue a desarrollar más el principio de cooperación, dándole tres objetivos principales:

2 Límites que suelen ser impuestos en todas las áreas que han sido tratadas por las Naciones Unidas, gracias a las bases y objetivos que tiene esta organización internacional. 
1. Promover el desarrollo de la ciencia y la tecnología espaciales y de sus aplicaciones;

2. Fomentar el desarrollo de una capacidad espacial pertinente y suficiente en los Estados interesados;

3. Facilitar el intercambio de conocimientos y tecnología entre los Estados, sobre una base mutuamente aceptable.

Finalmente, la declaración hace énfasis en la importancia de la cooperación en la Comisión sobre la Utilización del Espacio Ultraterrestre con Fines pacíficos y el Programa de las Naciones Unidas de aplicación de la tecnología espacial, puesto que por medio de la cooperación internacional los resultados del uso y la exploración del espacio ultraterrestre pueden ser aún mayores, especialmente porque estos pueden llegar a ayudar con los objetivos de desarrollo de múltiples países.

\section{La exploración en el espacio ultraterrestre bajo el principio de cooperación}

El desarrollo de la tecnología ha permitido que sea posible la exploración espacial, pero para lograr esto se necesitan un gran número de recursos. Por este motivo, a lo largo de la historia se han venido dando situaciones en las que diferentes países han colaborado en proyectos de exploración del espacio ultraterrestre. El primer ejemplo de lo ya descrito fue la misión ApoloSoyuz en 1975, que buscaba crear un ambiente de paz entre las naciones, por medio del primer “apretón de manos" en el espacio (siendo esto un símbolo de esperanza para la paz) (Rumerman). La misión se caracterizó por ser una de las primeras aproximaciones con fines pacíficos entre los Estados Unidos de América y la Unión Soviética, en plena Guerra Fría. Pero más allá del significado político de la misión, fue evidente la importancia frente a los avances técnicos que permitió este tipo de cooperación, como la creación de un sistema de acoplamiento para las naves soviéticas y norteamericanas. A su vez el programa llevó a que las dos naciones conocieran los proyectos del espacio ultraterrestre de cada uno y a que se llevaran capacitaciones en conjunto.

En 1973, se dio el siguiente evento en el que por medio de la cooperación internacional se pudo expandir el uso y la exploración del espacio ultraterrestre. Esto sucedió bajo la creación y utilización del Spacelab, el cual fue resultado de un acuerdo entre la NASA ${ }^{3}$ y la ESRO (hoy en día ESA). ${ }^{4}$ De acuerdo con este documento, la Agencia Espacial Europea se comprometió a dar un laboratorio útil en el espacio exterior a la NASA, la cual a cambio permitió que astronautas europeos fueran parte de la tripulación de vuelo de los transbordadores norteamericanos. Adicionalmente, diferentes experimentos y trabajos conjuntos se llevaron a cabo en este laboratorio por los europeos y los estadounidenses. Posteriormente, en 1998, el Spacelab dejó de ser utilizado, gracias a que el trabajo científico

3 Agencia del espacio del gobierno de Estados Unidos de América: Administración Nacional de la Aeronáutica y del Espacio.

$4 \quad$ ESA: siglas en inglés para la Agencia Espacial Europea. 
se debería llevar a cabo en la Estación Espacial Internacional.

El siguiente hito histórico, referente a la cooperación internacional y la exploración del espacio ultraterrestre, fue el Tratado Bilateral entre los Estados Unidos y la Unión Soviética, de 1977. En este, las dos naciones se comprometieron a cooperar en diferentes campos como la "ciencia y las aplicaciones especiales como la meteorología; el estudio del medio natural; la exploración del espacio cercano a la Tierra; la Luna y los planetas; la biología y la medicina espaciales, entre otros" (Tratado Bilateral entre los Estados Unidos y la Unión Soviética, 1977). También se comprometieron a entregar información útil para la exploración del espacio, y además a la creación de grupos conjuntos de trabajo y a cooperar en el área de los "vuelos tripulados con fines científicos y prácticos".

En 1990 fue puesto en órbita el telescopio espacial Hubble, logrando así la concretización de un proyecto conjunto entre la NASA y la ESA. Con este telescopio espacial se ha logrado un gran avance en la exploración del espacio, y de hecho se presume que

desde que fue puesto en órbita en 1990 para eludir la distorsión de la atmósfera -históricamente, el problema de todos los telescopios terrestres-, el Hubble ha permitido a los científicos ver el universo con una claridad jamás lograda. Con sus observaciones, los astrónomos confirmaron la existencia de los agujeros negros, aclararon ideas sobre el nacimiento del universo en una gran explosión, el big bang, ocurrida hace unos 13700 mi- llones de años, y revelaron nuevas galaxias y sistemas en los rincones más recónditos del cosmos. El Hubble también ayudó a los científicos a establecer que el Sistema Solar es mucho más joven que el universo. (El telescopio espacial Hubble)

Este fue el primer telescopio espacial del programa de la NASA de los Grandes Observatorios, ${ }^{5}$ programa en el que han participado la ESA y la JAXA, ${ }^{6}$ siendo uno de los ejemplos de cooperación en la exploración del espacio ultraterrestre más exitoso.

Entre 1986 y 1995 se desarrollaron los más claros ejemplos de colaboración internacional frente a la exploración, por medio de la creación y uso de la Estación Espacial Internacional y de la MIR. Estos ejemplos los explicaremos más a fondo en el punto $\mathrm{E}$ del presente trabajo.

Otros casos famosos en los que se evidencia el principio de cooperación en acción para la exploración y el uso del espacio ultraterrestre, ha sido la misión Stereo y la Swift. La segunda fue resultado de la cooperación del Reino Unido, Estados Unidos e Italia. En 2004, el observatorio espacial Swift fue lanzado al espacio ultraterrestre, y desde ese momento fue controlado por el ASI, ${ }^{7}$ con el fin de poder estudiar los rayos gamma, ultravioleta y rayos $X$ (UK Swift Scien-

\footnotetext{
5 El programa de los Grandes Observatorios tiene como fin la observación de determinadas regiones del espectro electromagnético, por medio de cuatro telescopios espaciales.

6 JAXA: siglas en japonés de la Agencia Japonesa de Exploración Aeroespacial.

7 ASI: siglas en italiano para la Agencia Espacial Italiana.
} 
ce Data Centre, 2014). La misión Stereo se dio gracias a la colaboración entre los Estados Unidos y el Reino Unido. Consistió en el envío en el 2006 de dos sondas a una órbita heliocéntrica, con el fin de observar el sol y sus fenómenos, tales como las erupciones que este puede llegar a presentar.

Los casos de cooperación internacional son muchos y resaltan la importancia de este principio. Mencionarlos todos sería imposible por lo que procederemos a explicar de forma detallada solo el caso del MIR para ejemplificar cómo funciona el principio en la práctica.

\section{E. El ejemplo de la cooperación internacional en la estación e spacial internacional mir}

Para 1986, ya habiendo visitado el espacio en múltiples ocasiones, Rusia crea un plan ambicioso y muy complicado. La exploración que hasta el momento se tenía del espacio ultraterrestre no había sido suficiente, motivo por el cual se desarrolló el proyecto MIR (siglas en ruso). La idea era un gran desafío, tanto tecnológico como científico, que iba a generar enormes costos. Sin embargo, nada de esto fue un impedimento para embarcarse en semejante odisea.

La construcción de la MIR comenzó en la Tierra pero se hizo en gran medida en el espacio. La partes que la conformaban debían enviarse por separado al espacio ultraterrestre e irse ensamblando, debido a su enorme tamaño y peso. Luego de cuatro años de trabajo, se terminó lo que sería un "edifico" de diez pisos con un peso de aproximadamente 136 toneladas, pasando a la historia como el objeto más grande jamás enviado al espacio. Desde esta se producirían avances y descubrimientos sin precedentes que contenían información tecnológica, ecológica y de la superficie atmosférica, entre otros.

A pesar de su gran éxito, luego de doce años de trabajo la MIR empezó a enfrentar problemas que ponían en gran peligro su funcionamiento. Entonces, para 1994 y basados en el principio de cooperación, Estado Unidos emprendió un viaje para ayudar con los problemas que afectaban a la MIR. Se enviaron astronautas estadounidenses que trabajaron por más de cuatro años de la mano con los astronautas rusos para arreglar todas las fallas que se habían presentado, sobre todo en los computadores internos y en las cámaras de oxígeno.

Para 2001, la MIR perdió su vida útil, y las piezas que no se desintegraron en la atmósfera cayeron como una lluvia en el pacífico. La MIR dejó una enorme cantidad de hallazgos y descubrimientos científicos gracias a la ayuda y aportes de una serie de naciones que participaron en el proyecto (esto fue posible en gran medida por la desintegración del régimen comunista dentro de la Unión Soviética). La ayuda de Estados Unidos fue de vital importancia ya que le salvó la vida a la estación y creó precedentes de cooperación internacional que marcan una tendencia y lineamientos para el futuro.

Pero tal vez el ejemplo más significativo de cooperación internacional en la exploración del derecho del espacio ultraterrestre es la Estación 
Espacial Internacional (en adelante EEI). La estación empezó a ser ensamblada en 1998 (aunque el proyecto se empezara a desarrollar desde 1980), con el fin de crear un centro de investigación más avanzado que el Spacelab, que estuviera siempre en el espacio y funcionara como un remplazo de algunas estaciones como la MIR. Por este motivo, fue puesta en la órbita terrestre como una estación espacial permanente. En esta operan cinco agencias del espacio (la NASA, la ESA, la Agencia Espacial Federal Rusa, la Agencia Espacial Canadiense y la Agencia Japonesa de Exploración Espacial). Adicionalmente, hay otras agencias que han participado en diferentes misiones, gracias a acuerdos y contratos que tienen con estas cinco agencias principales. De esta manera, la agencia espacial italiana y la agencia espacial brasileña se han sumado en la utilización de esta estación.

La estación "supone una gran plataforma de investigación para diversos estudios científicos y tecnológicos: productos de fabricación espacial, astronomía, detección terrestre a distancia, mecánica de materiales y fluidos, desarrollo de plantas y animales, biotecnología (crecimiento de cristales de proteínas y cultivos celulares), epitaxia de haz molecular (ehm)" (un laboratorio en órbita). Actualmente, esta estación es el objeto creado por el hombre de mayor envergadura en el espacio ultraterrestre.

Respecto a los aspectos técnicos de la eei, cabe recalcar que, completando una vuelta a la tierra en 91 minutos, aproximadamente a unos $400 \mathrm{~km}$ (altura de la iss), tendrá vida útil hasta 2020. Esta estación ha sido visitada por 205 personas de dieciséis países, tiene una capacidad para seis tripulantes y desde su puesta en órbita siempre ha estado habitada por al menos dos astronautas (la eei ahora usa linux). El aporte de suministros y transporte está a cargo de la agencia espacial rusa. Desde su creación ha sido administrada, gestionada, mantenida y desarrollada por las ya mencionadas agencias espaciales, de diferentes países, siendo así, tal vez, el ejemplo más significativo de la cooperación internacional en cuestiones de la exploración del espacio ultraterrestre.

\section{CAPITULO II}

\section{A. Exploración en el espacio}

\section{Régimen jurídico}

Desde el inicio de la exploración en el espacio, de la creación y el desarrollo del derecho espacial se ha hablado de buscar la paz y mantenerla sobre todas las cosas. Con el propósito de hacerlo, se crearon tres principios rectores que gobernarían todas las situaciones, tratados y demás circunstancias relacionadas con el espacio, como ya se ha venido explicando a través del presente texto. Volvemos a recalcar que estos principios son: el uso pacífico, no apropiación y cooperación. El primero se entiende como el deber y obligación de mantener las relaciones siempre en paz y tranquilidad. Sin importar que hubiera conflictos, problemas o contingencias siempre debían resolverse de tal forma que no se pusiera en peligro la paz. El segundo principio es la no apropiación, entendiéndose el es- 
pacio como una zona en la que la propiedad no existe y en la que todas las naciones tienen el mismo derecho. El tercero y último es la cooperación mediante la cual se desarrolla la ayuda entre países.

Sumado a esto, es importante resaltar el artículo I del Tratado sobre los principios que deben regir las actividades de los Estados en la exploración y utilización del espacio ultraterrestre, incluso la Luna y otros cuerpos celestes, que dice:

La exploración y utilización del espacio ultraterrestre, incluso la Luna y otros cuerpos celestes, deberán hacerse en provecho y en interés de todos los países, sea cual fuere su grado de desarrollo económico y científico, e incumben a toda la humanidad. El espacio ultraterrestre, incluso la Luna y otros cuerpos celestes, estará abierto para su exploración y utilización a todos los Estados sin discriminación alguna en condiciones de igualdad y en conformidad con el derecho internacional, y habrá libertad de acceso a todas las regiones de los cuerpos celestes. EI espacio ultraterrestre, incluso la Luna y otros cuerpos celestes, estarán abiertos a la investigación científica, y los Estados facilitarán y fomentarán la cooperación internacional en dichas investigaciones. (Naciones Unidas, 2002)

Como se puede ver, este artículo reúne los tres principios rectores de la actividad espacial. Además, incluye o resalta la importancia de la igualdad de todos los países sin importar su nivel de desarrollo.

Desde 1967 se inició la enorme tarea de crear una serie de tratados que regularan la actividad espacial. Dichos tratados se crearon de la mano con la Comisión para la Utilización del Espacio Ultraterrestre con Fines Pacíficos (Copuos), creada en 1959, que además tenía dos subcomisiones, la jurídica y la científica. A diferencia de muchos otros, los tratados no se creaban y se imponían a los Estados, sino que se creaban por consenso. Lo que esto implica es que todos los Estados aceptan la decisión o por lo menos no se encuentran en un opuesto discordante. En consecuencia,

[...] la regla del consenso ha animado el compromiso [...] y promovido laboriosos esfuerzos entre los negociadores en orden a acomodar las diversas posturas. Los tratados se aceptarán más, pues todos los puntos de vista, incluidos los de las principales potencias espaciales, son tenidos en cuenta. En resumen, la regla del consenso es la técnica empleada por el Comité [...] para la acomodación de los diferentes intereses de los Estados miembros. (Jasentuliyana, p. 356)

De esta forma, se crea un sano ambiente de desarrollo, confianza y cooperación entre los países puesto que es claro que la unanimidad y consenso se da en beneficio de ellos mismos.

La enorme acogida y éxito del consenso provocó una buena productividad "legislativa", que para 1980 trajo el producto de cinco tratados históricos, entre otros: 1) 1966. Tratado sobre los principios que deben regir las actividades de los Estados en la exploración del espacio ultraterrestre, incluida la Luna y otros cuerpos celestes, 2) 1967. Acuerdo sobre salvamento y devolución de astronautas y la restitución de objetos lanzados al espacio ultraterrestre, 3) 
1971. Convención sobre la responsabilidad internacional de los daños causados por objetos espaciales, 4) 1974. Convenio sobre el registro de objetos lanzados al espacio ultraterrestre, 5) 1979. Acuerdo que debe regir las actividades de Ios Estados en la Luna y en otros cuerpos celestes. Esto mostró que el derecho del espacio ultraterrestre pasaba por lo que muchos doctrinantes denominan "la época dorada”, debido a la agilidad, producción de tratados y acuerdos de países que no solo respetaban las decisiones por consenso sino que le mostraban al ya ensangrentado mundo que con confianza, consenso y cooperación sí era posible tener un territorio de todos y para todos, en el que reinara la paz.

Sin embargo, con la llegada del siglo XXI se ha venido desacelerando la producción de la normativa espacial, y en gran medida por las implicaciones económicas que esto acarrea, el consenso parece estar perdiendo su eficacia y su carácter obligatorio y vinculante, y ya se pone en tela de juicio, dejando todo el derecho espacial en un limbo crítico que de no solucionarse podría llegar a generar catástrofes irremediables con graves resultados. Para entender mejor esto proseguiremos por hacer un análisis de la economía en relación con el espacio ultraterrestre.

\section{Análisis económico}

El inicio de la exploración del espacio por parte del hombre fue gracias a la tensión que se vivía en la Tierra y a las enormes ansias de las grandes potencias por ganar la guerra. No obstante, esto fue solo la chispa que inició la exploración y fue solo cuestión de tiempo para que el mundo entero reconociera la importancia del espacio ultraterrestre.

Las grandes potencias y principalmente la Unión Soviética y Estado Unidos fueron los grandes pioneros de la exploración espacial. No solo producían descubrimientos fascinantes sino que le demostraban al mundo su poder a través de constantes lanzamientos de cohetes y posicionamiento de satélites. Sin embargo, para el resto del mundo el espacio no era una opción. Era una nueva conquista del reducido número de países que tenían los suficientes recursos para acceder a este, mientras la gran mayoría observaba desde la Tierra lo que parecía imposible.

Con el pasar del tiempo la exploración del espacio y la explotación del mismo por parte del hombre se volvieron una realidad cada vez más accesible. Las dos potencias ya no eran las únicas capaces de acceder a este, sino que cada año nuevos países lograban llegar al tan anhelado espacio ultraterrestre. Entonces, el número de países participantes comenzó a crecer de forma alarmante, como lo muestra la siguiente gráfica (OECD, 2011): ${ }^{8}$

8 Permiso de cita del OCDEilibrary: OECD (2011), The Space Economy at a Glance, OECD Publishing.

doi: $10.1787 / 9789264111790-e n$ 


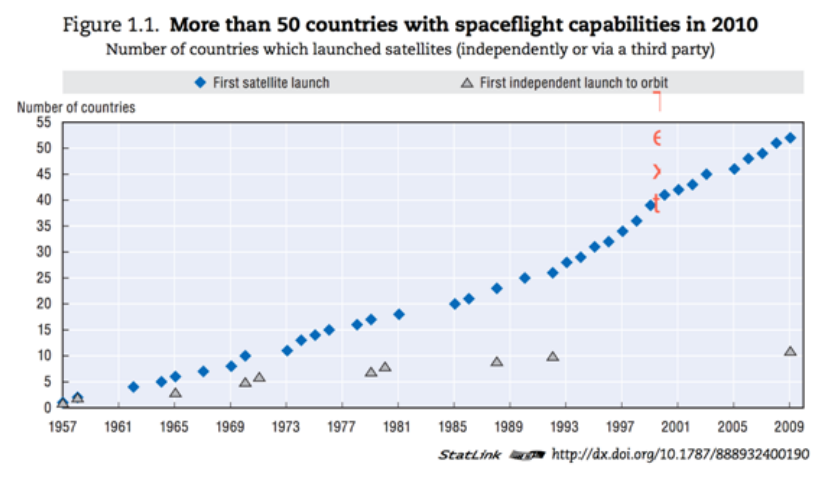

A mediados del siglo $\mathrm{XX}$, el número de países que podía acceder al espacio era menos de cinco. Pero fue tal el crecimiento y evolución de la exploración que para 2009 más de 53 países habían enviado por lo menos un satélite propio. Lo que esto significa es que con el pasar del tiempo cada vez más países lograrán llegar al espacio y cada año va a haber un mayor número de naciones que participarán en la exploración de este.

Este enorme crecimiento del mercado espacial se debe a la importancia y al valor agregado que recibe cada Estado por tener sus propios satélites. Esto no significa únicamente tener más tecnología o información, sino es también tener independencia con respecto a terceros países. Por ende, no es sorpresivo que ahora un sinnúmero de naciones tengan un presupuesto destinado a la exploración del espacio.

Luego de haber visto el enorme crecimiento de la participación de países en el espacio ultraterrestre, no resulta sorprendente que este sea un recurso valorable para una economía en crecimiento, en la cual se invierten miles de millones de dólares cada año. En la siguiente tabla podemos ver los presupuestos destinados a los proyectos espaciales por parte de los países del G20.

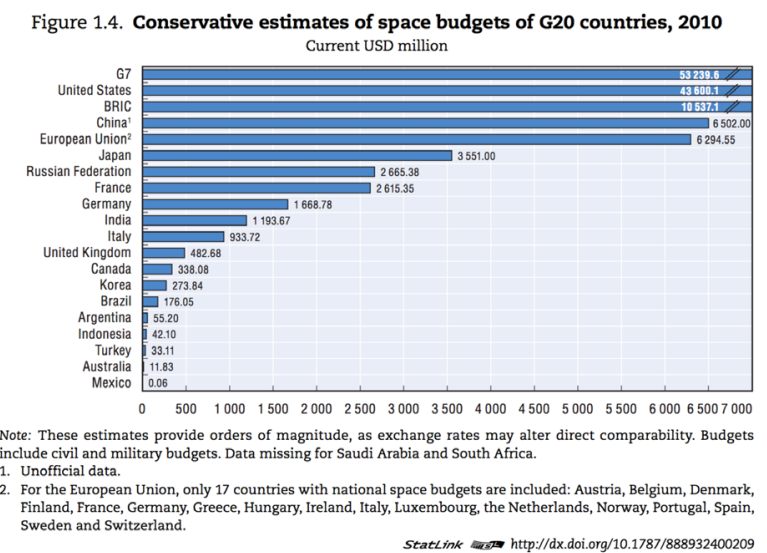

Como se puede apreciar, solo sumando los primeros tres puestos, la inversión en proyectos espaciales supera los 100000 millones de dólares. Por si esto no fuera lo suficientemente impresionante, cada año este número crece exponencialmente. Ahora, ¿por qué pasa esto?

Desde el descubrimiento de los beneficios que se pueden obtener a través del uso del espacio ultraterrestre en la tecnología, y en la vida diaria de las empresas y de las personas naturales, se ha hecho imposible la vida sin la explotación del espacio ultraterrestre.

Hoy en día, el espacio es vital para actividades como: navegación, comunicaciones, meteorología, observación, tecnología y datos, entre muchos otros. No solo son vitales para la vida humana, sino que si dejara de funcionar la mayor parte de la economía en el mundo enteró colapsaría instantáneamente. Más que cualquier otro sector de proyectos o inversiones es el espacio la economía con mayor crecimiento por la enorme incidencia e importancia que tiene en la vida diaria de todos los seres humanos. 
Es fácil entenderlo con la simple imagen de toda la tecnología que cada ser humano usa a diario (teléfono, internet, televisión), que de no tenerse sería casi imposible trabajar, estudiar o desarrollar

Sumado a esto, el espacio es un mercado que a pesar de sus altos costos es uno de los pocos que superó la crisis económica mundial de 2008. Podría decirse que esto se debe a que es un mercado necesario para todos los países. Es vital para las actividades de todos, por eso es tan importante como el agua o la misma comida. Por ende, no solo crece a una velocidad alarmante sino que también está bloqueado de cualquier eventualidad por la necesidad e importancia que tiene en el mundo moderno.

Resulta entonces obvio cómo la inversión privada está íntimamente relacionado con éste mercado. Dicha intervención de las empresas privadas comerciales ${ }^{9}$ no solo los involucra como patrocinadores sino como los mismos prestadores de los servicios. En consecuencia, se puede ver cómo: "En este contexto, hay un enorme movimiento de los países con actividades en el espacio de pasar del sistema regulador de actividades espaciales basado en tratados hacia un régimen enfocado en sistemas nacionales comerciales" (OECD, p. 32, traducción libre). Esto se debe a que con el enorme crecimiento de las actividades espaciales, los tratados generados en los ochenta ya no son suficientes. Por ende, los países se han visto en la obligación de crear

9 Unos ejemplos son Virgin Galactic, Space x, Bigelow y Orbital Sciences. sus propios regímenes con el fin de suplir los vacíos que el derecho del espacio ha dejado.

A continuación podemos ver la gráfica del crecimiento de las leyes nacionales con respecto a los asuntos espaciales:

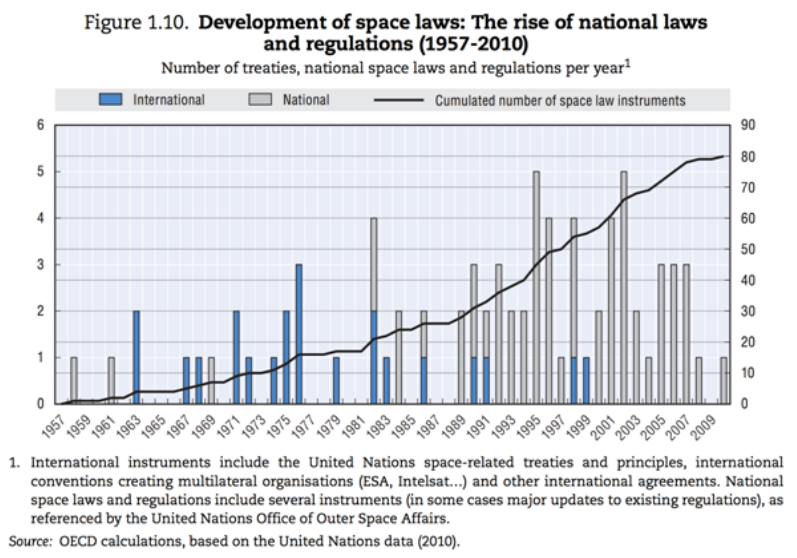

Podemos ver que en el siglo XX el crecimiento y uso del derecho espacial con base en los tratados era casi total y todas las naciones funcionaban con base en este. No obstante, desde 1989 se volvió clara la preferencia de los países de aplicar las reglas nacionales y de basarse solo en estas. Es tan extremo que desde el año 2000 todo se ha hecho con base en las reglas nacionales creadas por cada país, afectando así la misma colaboración internacional y por ende la misma exploración del espacio ultraterrestre.

Surge entonces la pregunta sobre si al ser el negocio del espacio tan próspero y vital para la vida humana está siendo efectivamente regulado por el derecho internacional. Con el fin de resolver este interrogantes, en el acápite siguiente plantearemos lo que consideramos son los problemas del derecho internacional del espacio y las posibles soluciones a estos. 


\section{Problemas del derecho del espacio en el siglo xxi}

Desde el inicio, el derecho del espacio sorprendió por su eficacia basada en plena confianza, consenso y cooperaciones de los países. Con su aplicación, y la de los principios rectores de uso pacífico, no apropiación y cooperación, se logró demostrar que era posible conservar una zona en donde todos pudieran participar por igual sin que hubiera lugar a conflictos o guerras posteriores.

Como ya se mencionó, la "época dorada" del derecho del espacio sobrepasó cualquier expectativa y demostró que lo que parecía imposible no lo era y con la voluntad de todos podría mantenerse la paz y la justicia. No obstante, el mundo de las tecnologías ha evoluciona a una velocidad inmensurable, permitiendo la posibilidad de la explotación del espacio ultraterrestre. Por ende, los países y las mismas empresas privadas luchan día a día por tener acceso a este, para usarlo económicamente, generando así un auge en el que todos los países quieren participar y sacarle mayor provecho. En consecuencia, las intenciones de cada uno de los Estados empiezan a primar por encima del interés general, poniendo en jaque al derecho consensual del espacio por ser dispositivo y flexible.

Aunque son muchos los problemas que se podría encontrar en el derecho del espacio, a continuación nos enfocaremos en los tres que ponen en mayor peligro al sistema: 1) Consenso; 2) Conflicto de intereses y 3) Disposición.

\section{Consenso}

Como ya se mencionó, con la creación del Copuos se decidió que todas las decisiones deberían tomarse por consenso. Esto ha sido un enorme éxito debido al compromiso y confianza que se ha forjado entre naciones. A pesar de esto, hoy en día el consenso parece no ser suficiente.

Desafortunadamente, algunos países han abusado de la figura del consenso, desnaturalizándola. Dicho abuso se presenta debido a que los países que no están de acuerdo con alguna decisión prefieren no adherirse a un tratado que los puede llegar a afectar ahora, o en un futuro. Al obstaculizar estos procesos por medio de la abstención negativa, el consenso como tal no necesariamente se debería ver afectado, pero la práctica excesiva de la negativa, desfigura y previene cualquier resultado positivo que se podría esperar.

Como es de público conocimiento, hay ciertos temas sobre los cuales aún no se ha podido llegar a un consenso, como por ejemplo el límite del espacio aéreo y la responsabilidad por los desechos espaciales. Sumado a esto, temas como la energía nuclear no han sido plasmados en un acuerdo, y llegar a un consenso parece imposible.

Surge entonces el enorme problema, y así la duda: ¿debemos dejar desacelerar la producción jurídica del derecho del espacio, por respetar sobre todas las cosas el consenso? 
Para nosotros, la respuesta resulta evidente. Aunque entendemos que el consenso es la base de la confianza, compromiso y cooperación, consideramos que la figura es tan flexible que logró desnaturalizarse por medio del abuso de la misma. Los países han descubierto que participar en el consenso no es obligatorio, en cambio, si aceptaban las decisiones iban a imponerse restricciones que los podrían afectar en un futuro. Entonces, preguntamos: ¿qué país tomaría decisiones que le generan restricciones si el hecho de no tomarlas no le genera perjuicios?

En un ensayo de la Universidad de Berkeley, se explica el funcionamiento del consenso:

En el marco de la creación de reglas multilaterales, las negociaciones por consenso no significan más que la ausencia de un rechazo formal a una decisión particular. Esto no significa que haya el apoyo positivo necesario para una aprobación del tratado por parte de los órganos responsables de la ratificación. En ausencia del apoyo positivo el consenso puede que no guíe a la ratificación por parte de cada Estado ya que cada uno decide si le conviene o no hacerlo. (Danilenko, traducción libre)

Como podemos ver, el consenso no implica una ratificación por parte de los Estados, por lo que pueden estos entrar a decidir si quieren ratificar el tratado o no, según sus propios intereses.

Por estos motivos, tratados como el de la Luna no ha sido ratificado por la mayoría de países, generando una enorme desaceleración de la efectividad del derecho del espacio, ya que cada vez menos países ratifican tratados, dejándolos solo como normas de papel. Además de esto, llegar al consenso es cada vez más difícil, puesto que los Estados no quieren verse amarrados por más normas o controles que les puedan impedir o afectar sus intereses económicos.

\section{Conflicto de interés}

Antiguamente, los países que realmente podían acceder al espacio, por el alto costo que esto implicaba, eran muy pocos, y eran especialmente las grandes potencias económicas. No obstante, la investigación y tecnología han permitido que innumerables proyectos se puedan concretar, haciendo que cada vez sea más posible el acceso de todos al espacio. Además de esto, el uso del espacio es hoy tan frecuente que sin este muchos proyectos serían imposibles. Los satélites son el mejor ejemplo de esto, puesto que son instrumentos que se convirtieron en elementos fundamentales para el día a día de actividades, desde educativas hasta de control climático. Por ende, las empresas tanto públicas como privadas se han encargado de invertir millonarias cifras, para llevar más y más objetos al espacio, por ser estos tremendamente rentables.

Aunque esto es bueno para la economía y los mismos países en desarrollo, el alto crecimiento económico e inversión espacial son los principales causantes de lo que consideramos el segundo problema del derecho del espacial, que es el conflicto de interés.

Llegar al consenso en la "época dorada" era más fácil debido a la poca relación o intención 
que se tenía en llegar al espacio. Los países aceptaban y ratificaban tratados porque encontraban en ellos unos controles eficientes que no los afectarían. No obstante, hoy en día vemos un gran cambio en el que la gran mayoría de países ya han participado en el derecho del espacio o por lo menos tiene una intención cercana de hacerlo. En consecuencia, cada país entra a ser parte del mercado y por ende a tener intereses propios y legítimos que nublan su objetividad.

Pero el hecho de que ahora todos los países son inversionistas o potenciales inversionistas sesga su posición y les quita la posibilidad de tomar medidas imparciales y justas. Por ende, en situaciones en las que se pretenden tomar decisiones por consenso, no se está pensando en el bien mundial o en el respeto de los tres principios sobre todas las cosas, sino en sus propios intereses, y es aquí donde se genera un conflicto. ¿Cómo puede un país buscar el interés mundial cuando su interés nacional prima?

En nuestro concepto, este conflicto de interés y el ya mencionado caos del consenso ponen en jaque el derecho del espacio basado en la cooperación y confianza. Esto se debe a que los principios se quiebran cuando los intereses nacionales empiezan a primar sobre los universales, ya que se pierde la imparcialidad y se busca la mayor cantidad de beneficios nacionales $\sin$ importar las necesidades de los demás.

\section{Disposición}

Uno de los mayores problemas del derecho internacional es la vinculación u obligatoriedad que este genera. Aunque se puede extender por medio del soft law, continúa teniendo problemas. Consideramos que esto se debe, principalmente, a la falta de control o sanciones que obliguen a los países a acatarlos.

Como ya se dijo en reiteradas ocasiones, entendemos la necesidad de la confianza y la cooperación que debe reinar en el derecho del espacio. Sin embargo, y enlazado con los problemas de consenso y de conflictos de interés ya mencionados, consideramos que la disposición es tremendamente peligrosa para el derecho espacial.

En un principio, acatar el derecho del espacio era simple, puesto que eran pocos los que realmente accedían a este, pero el panorama hoy es diferente. Al haber un gran número de países que quieren o que ya son parte de los participantes en el espacio, darles la posibilidad de elegir o no el ratificar tratados es para nosotros un arma de doble filo. Por un lado, les da a los países la posibilidad de tomar decisiones libremente y acatar el derecho del espacio, no porque se los impusieron, sino porque ellos así lo consideraron y dispusieron. Por otro lado está el conflicto de intereses que evita que tomen decisiones desfavorecedoras, optando por la no ratificación de los tratados, puesto que hacerlo solo les generaría ciertos controles y ningún beneficio.

En suma, el hecho de que el derecho del espacio sea dispositivo genera cooperación y confianza. Sin embargo, en un mundo donde existen conflictos de interés, suelen primar otras prioridades por encima del deber de cooperación y confianza. Entonces, y al no haber ningún tipo 
de sanción o control claro, pasaría el derecho espacial a un segundo plano, sin que haya ningún tipo de sanción, puesto que al fin y al cabo no es más que un derecho dispositivo.

\section{B. Propuesta. Soluciones para la crisis del derecho del espacio}

Luego de haber expuesto los problemas del derecho del espacio y la exploración, procederemos a proponer las posibles soluciones, así: 1) Reforma al consenso, 2) Fondo del espacio y 3) Subcomisión verificadora.

\section{Reforma al consenso}

Teniendo en cuenta que la figura del consenso se ha desnaturalizado por su abuso, consideramos que esta debe reformarse y volverse más estricta. Para esto, pretendemos hacer una reforma del Copuos, en cuanto a sus posibles funciones y a su misma estructura.

En primer lugar, proponemos la reforma de la subcomisión legislativa, de manera tal que se componga por un número impar de abogados expertos en la materia del derecho del espacio ultraterrestre, que deberán ser elegidos por el presidente de la ONU con base en un sistema meritocrático. Estos deberán ser siempre asesorados por la Subcomisión de Asuntos Científicos y Técnicos.

En segundo lugar, la subcomisión legislativa tendrá el deber de estudiar los problemas actuales y con base en estos presentar una iniciativa legislativa, si los Estados aún no lo han hecho. Luego, los países tendrán un año prorrogable por un período igual para llegar al consenso sobre la decisión, resolución o tratado que solucione el problema planteado por la subcomisión. En caso de que esto no se logre en este período de tiempo, el Copuos, en conjunto con la UIT (Unión Internacional de Telecomunicación), podrán imponer una serie de sanciones a los países que estén bloqueando los procesos de consenso sin proponer unas soluciones viables a las causas que tranquen estas iniciativas legislativas. Entre las sanciones que se podrían considerar, está la de limitar el acceso a las frecuencias y orbitas.

\section{Fondo del espacio}

El espacio y algunas zonas de este, como la órbita de los satélites geoestacionarios, son recursos naturales limitados, por lo tanto deben cuidarse y respetarse..$^{10}$ Sin embargo, debido al enorme número de objetos espaciales lanzados, la cantidad de desechos en el espacio es cada vez más grande. Esta contaminación pone en peligro a los otros objetos espaciales enviados por el hombre y por ende a la misma exploración del espacio ultraterrestre.

Por esto, nuestra segunda propuesta es de carácter económico. Pretendemos proponer la creación de un fondo que recaude dinero para proteger el espacio. Entonces, cada empresa pública o privada que tenga control o sea dueña de alguno de estos objetos ubicados en el espacio ultraterrestre deberá pagar el $5 \%$ de

10 Artículo 33 del Tratado de Málaga. 
las ganancias que recibe por el uso de estos objetos. La única excepción que por el momento vislumbramos es frente a los objetos estatales con fines de interés general, los cuales quedarán exentos de dichos cobro, pero deberán pagar un derecho de acceso que será del $3 \%$ del costo del lanzamiento del objeto espacial, si ya han tenido previamente acceso a este tipo de tecnologías.

El dinero será administrado por el Copuos, y deberá ser dirigido a la investigación y limpieza de desechos humanos en el espacio ultraterrestre. Invertirán además en tecnología para proteger el espacio, extendiendo así la cooperación internacional en el espacio.

\section{Subcomisión verificadora}

Debido al auge en el uso del espacio, los conflictos y el desacato de las normas internacionales deben tener un control estricto que limite 0 prevenga que los Estados o los privados actúen en contra de la legislación existente. En caso de hacerlo, deberán ser arduamente castigados, puesto que el espacio ultraterrestre debe usarse de manera pacífica y bajo el interés de la humanidad entera.

Nuestra última propuesta se basa en crear la cuarta subcomisión del Copuos, que se llamaría la Subcomisión Verificadora. Estaría compuesta por nueve abogados espaciales, elegidos por mayoría absoluta en la sala plena. Esta subcomisión tendría dos deberes principales, que son: dirimir conflictos entre Estados relacionados con el derecho del espacio y sancionar a los
Estados que no acataron normas del derecho del espacio. Esto lo harían con la ayuda técnica y científica necesaria, dada por la Subcomisión de Asuntos Científicos y Técnicos.

Este nuevo órgano funcionaría como un tribunal de arbitramento y tendría tres salas compuestas por tres magistrados cada una. En cuanto a su regulación, deberá definirla la sala plena o en su defecto la subcomisión legislativa. Las decisiones que las salas tomarían serían de carácter obligatorio y deberán acatarse de manera inmediata. Sin embargo, creemos necesario que haya un sistema de apelación de las decisiones, que se deberán hacer frente a todos los magistrados de esta subcomisión. Aunque es discreción de las salas, las decisiones que tomen estas podrán contener sanciones de carácter económico, político o restrictivo, de acuerdo con la falta cometida.

La creación de esta subcomisión es de vital importancia por la amplia impunidad que pueden llegar a tener los Estados frente a la infracción del derecho del espacio. La posibilidad de no acatar los tratados es muy alta, y en el espacio, en donde el poder y las ventajas que se pueden obtener son tan grandes, esto no se puede permitir. Por ende creemos que, por el bien universal y de los mismos países, debe crearse esta subcomisión que supervise y verifique el cumplimiento de los tratados. 


\section{CONCLUSIONES}

Luego de varias guerras y mucha sangre derramada, los Estados buscaban a toda costa mantener la paz. Como en la Tierra ya no era posible empezar de nuevo, se llegó al espacio y allí se encontró la esperanza y posibilidad de cambio. Se creyó que el espacio podía ser la zona en donde todo fuera de todos, todo se regiría por la paz, y la ayuda de unos con otros era la clave de todo. Por esto se creó en 1967 la Constitución del Espacio, que desarrolló los tres principios rectores de todo lo relacionado con el espacio: uso pacífico, cooperación y no apropiación.

Aunque todos fueron de vital importancia, la cooperación fue crucial para el desarrollo del espacio y para la misma evolución. Acompañada de la confianza, compromiso y consenso, trajeron en la segunda mitad del siglo XX lo que sería la "época dorada" del derecho del espacio. Se dieron grandes avances tecnológicos, y con la ayuda y cooperación de todos, algunos países lograron llegar al espacio. La cooperación entre naciones, en proyectos como el del telescopio Hubble, el Spacelab, la MIR o la Estación Espacial Internacional, nos ha permitido desarrollar no solo la tecnología con la que vivimos todos los días, sino que adicionalmente nos ha dejado explorar el espacio, ampliando nuestros propios límites físicos y científicos. Esto, a su vez, ha conllevado un proceso legislativo en el que la cooperación se ha venido perfeccionando. A pesar de esto, la normativa existente no garantiza el uso adecuado del espacio ultraterrestre, y la sola cooperación no es suficiente para man- tener el uso pacífico de este lugar afuera de la Tierra.

Tristemente, la "época dorada" acabó, y lo que aparenta ser una desaceleración en la creación legislativa del derecho del espacio, nos ha llevado poco a poco a una futura crisis. El abuso del derecho y la desnaturalización han minado la tan exitosa figura del consenso. No siendo poco, los avances tecnológicos terminaron por poner los intereses nacionales por encima de los universales, permitiéndoles desacatar y abandonar la ley del espacio, sin mayores repercusiones. Como es evidente, no debería disminuir la creación de legislación del espacio, sino, por el contrario, debería tener prioridad y salir adelante. Por eso nos encontramos en un punto crítico, en donde el derecho del espacio se quedó corto, lo cual contrasta con la inmensidad de posibilidades que nos ofrece el uso y exploración del espacio ultraterrestre.

Por ende, proponemos la reforma del Copuos, con el fin de evitar una eminente tragedia, alterando la forma en la que se legisla y en la que se controla y verifica el uso adecuado del espacio ultraterrestre. Proponemos la modificación del consenso y la aplicación efectiva de los tratados, bajo la amenaza de sanciones económicas y/o políticas a los Estados o entidades privadas. Por último, proponemos la creación de un fondo que reciba dineros en calidad de impuestos para cuidar el espacio.

El futuro está en el espacio ultraterrestre, pero no podemos cometer los mismos errores que ya cometimos en la Tierra. Debemos aprender 
y asegurar el cumplimiento de las normas, para que allí tengamos la tan anhelada paz y justicia, no en provecho de solo algunos, sino de todos.

\section{Referencias}

Acuerdo que debe regir las actividades de los Estados en la Luna y otros cuerpos celestes (1966). Recuperado de http://www.dipublico. com.ar/instrumentos/54.html

Altura de la ISS. Recuperado dehttp://www. heavens-above.com/issheight.aspx?lat $=0 \&$ In $\mathrm{g}=0 \&$ alt $=0 \& \mid \mathrm{loc}=$ Unspecified $\& T Z=C E T$

Danilenko. Outer Space and the Multilateral Treaty-Making Process. Recuperado dehttp:// www.law.berkeley.edu/journals/btlj/articles/ vol4/Danilenko/HTML/text.html.

Declaración sobre la cooperación internacional en la exploración y utilización del espacio ultraterrestre en beneficio e interés de todos los Estados, teniendo especialmente en cuenta las necesidades de los países en desarrollo (1996). Recuperado de: http://www.oosa. unvienna.org/pdf/publications/STSPACE11S. pdf. P.71

El telescopio espacial Hubble. Recuperado de: http://www.astromia.com/astronomia/telescopiohubble.htm

La EEl (Estación Espacial Internacional) ahora usa Linux. Recuperado de: http://www. ucontrol.com.ar/forosmf/off-topic/la-eei\%28estacion-espacial-internacional\%29-ahora-usa-linux/?wap2
OECD (2011). The Space Sector in 2011 and Beyond. The Space Economy at a Glance, 2011. OECD Publishing. Recuperado de: http:// dx.doi.org/10.1787/9789264113565-5-en

Principios que han de regir la utilización por los Estados de satélites artificiales de la Tierra para las transmisiones internacionales directas por televisión (1982). Recuperado de: http://www.oosa.unvienna.org/pdf/publications/STSPACE11S.pdf

¿Qué es? Recuperado de: http://www.urosario.edu.co/Internacionalizacion/Oportunidades-Cooperacion-Internacional/Que-es/

Resolución 1721 A y B (XVI) de la Asamblea General, de 20 de diciembre de 1961. Recuperado de: http://www.oosa.unvienna.org/pdf/ gares/ARES_29_3234S.pdf

Rumerman. Human Space Flight: a Record of Achievement, 1961-1998.

The Swift Gamma-Ray Burst Mission (2014). Recuperado de: http://www.swift.ac.uk/

Tratado Bilateral entre los Estados Unidos y la Unión Soviética (1977). Recuperado de: http://biblio.juridicas.unam.mx/libros/2/947/8.pdf

Tratados y principios de la Naciones Unidas sobre el espacio ultraterrestre (2002). Publicación de las Naciones Unidas. Recuperado de: Ihttp://www.oosa.unvienna.org/pdf/publications/STSPACE11S.pdf 
Seara (1981). Derecho y política en el espacio cósmico.

Un laboratorio en órbita. Recuperado de: http:// www.estacionespacial.com/ciencia.php

Valera, V. (2013). La importancia de fomentar la cooperación internacional en la utilización y exploración del espacio ultraterrestre. Revista de Derecho, Comunicaciones y Nuevas Tecnologías, 10. Universidad de los Andes (Colombia).

Williams (1990). Derecho Internacional Contemporáneo. 